\section{Leggi qui}
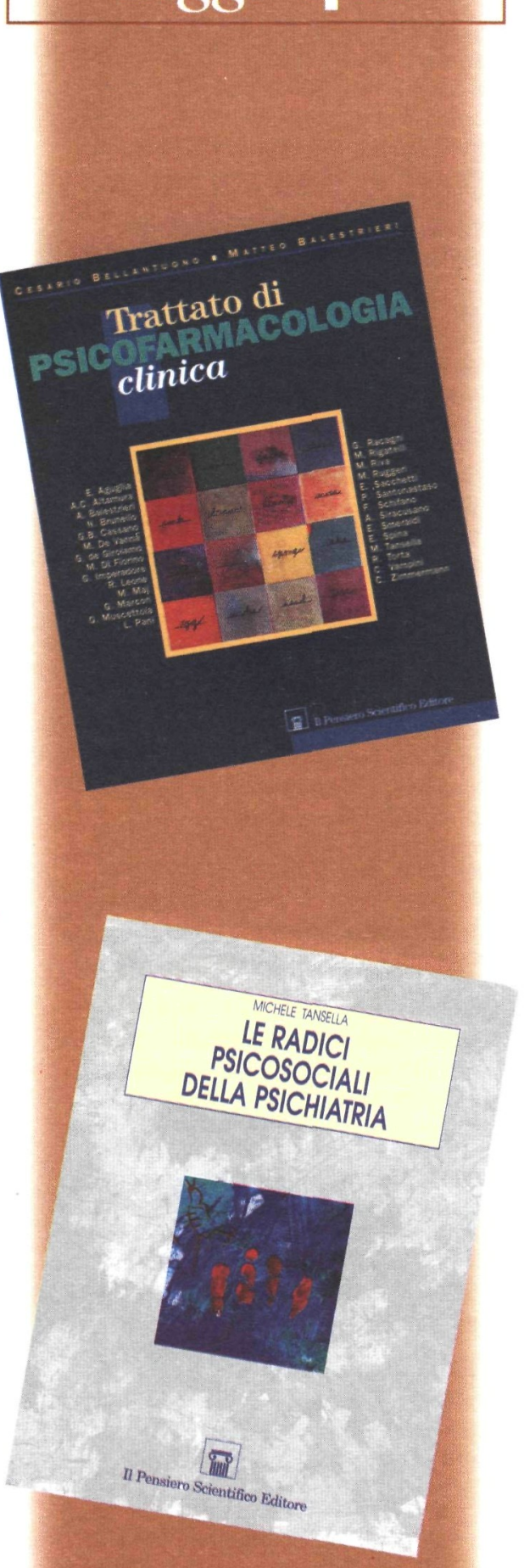

\section{Assistenza e Ricerca
in Salute Mentale \\ Cesario Bellantuono, Matteo Balestrieri Trattato di psicofarmacologia clinica}

1104 pagine. $€ 95,00$

I recenti progressi delle neuroscienze hanno aperto nuove possibilità terapeutiche anche nel campo della neuropsicofarmacologica clinica, in particolare per quanto riguarda la sintesi di nuove molecole caratterizzate da maggiore efficacia terapeutica e minori effetti collaterali.

Questo nuovo trattato di psicofarmacologia clinica, curato da Cesario Bellantuono e Matteo Balestrieri - che si avvale della collaborazione dei più eminenti psichiatri e psicofarmacologi italiani, quali, fra gli altri, Eugenio Aguglia, Alfredo Altamura, Giovanni Cassano, Mario Maj, Giovanni Muscettola, Luca Pani, Giorgio Racagni, Emilio Sacchetti, Alberto Siracusano, Enrico Smeraldi, Michele Tansella e Riccardo Torta - non può non tener conto di tali progressi.

Vuole offrire un'occasione di aggiornamento scientifico e culturale alla classe medica italiana, si rivolge non solo agli psichiatri, ma anche ai neurologi, ai geriatri, ai medici di medicina generale ed a tutti gli specialisti che nella loro pratica clinica utilizzano questi farmaci o vogliono aumentare le loro conoscenze nel campo della psicofarmacologia clinica.

Michele Tansella

\section{Le radici psicosociali della psichiatria}

274 pagine. $€ 30,00$

In questo volume sono pubblicati i lavori più importanti apparsi durante un lungo periodo, caratterizzato da una produzione scientifica di alto profilo.

Michele Tansella ha più di ogni altro contribuito a dare una reputazione internazionale alla psichiatria sociale italiana, tanto che Verona e il suo modello di assistenza sono ora conosciuti in tutto il mondo.

Egli è uno dei principali sostenitori della necessità di sviluppare i Servizi di salute mentale nella comunità e di utilizzare un approccio psicosociale al trattamento dei disturbi mentali.

La varietà dei temi indagati nei sei filoni nei quali sono stati suddivisi i suoi 20 lavori pubblicati in questo volume, dà un'idea della vastità del suo approccio.

Dalla Presentazione di Sir David Goldberg

\author{
Vuoi leggere di questi libri? \\ Acquistali. Clicca su www.pensiero.it \\ È così facile...
}




\section{EvidenteMente?}

Con scritti di Fonagy, Geddes, Goldberg, Huxley, Roth, Tansella, Thornicroft, Williams

Il Pensiero Scientifico Editore www.pensiero.it www.vapensiero.info

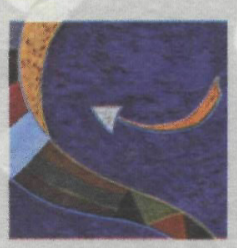




\title{
Invited Papers
}

91
Contemporary thinking about the role of genes
and environment in eating disorders
C.M. Bulik, F. Tozzi

Schizophrenia: still Kraepelin's Dementia Praecox?

H. Häfner

113

Cannabis use and risk of psychosis: an etiological link?

H. Verdoux, M. Tournier

\section{Original Papers}

\begin{abstract}
of dysthymic patients in Italian primary care C. Barbui, L. Garattini, I. Krulichova, G. Apolone on behalf of the Study Group DYSCO, E. Negri (Data Management), E. Ricci (Data Analysis)
\end{abstract}

L'accreditamento del Dipartimento di Salute Mentale. Criteri di classificazione e di valutazione G. Grassi 